CRYSTALLOGRAPHIC COMMUNICATIONS

ISSN 2056-9890

Received 12 May 2017

Accepted 6 June 2017

Edited by M. Weil, Vienna University of Technology, Austria

Keywords: Technetium; sodium; redetermination; crystal structure; low temperature; high precision.

CCDC references: 1554512; 1554511

Supporting information: this article has supporting information at journals.iucr.org/e

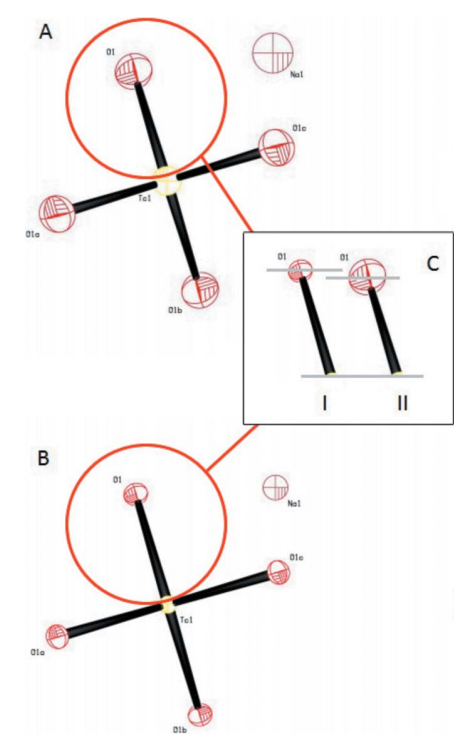

OPEN $\odot$ ACCESS

\section{Redetermination of the crystal structure of $\mathrm{NaTcO}_{4}$ at 100 and $296 \mathrm{~K}$ based on single-crystal X-ray data}

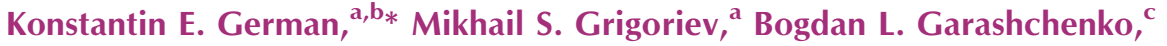 \\ Alexander V. Kopytin ${ }^{a, d}$ and Ekaterina A. Tyupina ${ }^{c, e}$ \\ ${ }^{\mathbf{a}}$ A.N. Frumkin Institute of Physical Chemistry and Electrochemistry, Russian Academy of Sciences, 31 Leninsky prospekt,
119071 Moscow, Russian Federation, ${ }^{\mathbf{b}}$ Medical University Reaviz, 2 Krasnobogatyrskaya, building 2, 107564 Moscow,
Russian Federation, ${ }^{\mathbf{c}}$ D. Mendeleyev University of Chemical Technology of Russia, 9 Miusskaya pl., 125047 Moscow,
Russian Federation, ${ }^{\mathbf{d}}$ Kurnakov Institute of General and Inorganic Chemistry, Russian Academy of Sciences, 31 Leninsky
prospect, 119991 Moscow, Russian Federation, and ${ }^{\mathbf{e}}$ National Research Nuclear University, 31 Kashirskoye sh., 115409
Moscow, Russian Federation. ${ }^{*}$ Correspondence e-mail: guerman_k@mail.ru
}

The redetermination of the title compound, sodium pertechnate, from singlecrystal CCD data recorded both at 296 and $100 \mathrm{~K}$ confirms previous studies based on X-ray powder diffraction film data [Schwochau (1962). Z. Naturforsch. Teil A, 17, 630; Keller \& Kanellakopulos (1963). Radiochim. Acta, 1, 107-108] and neutron powder diffraction data using the Rietveld method [Weaver et al. (2017). Inorg. Chem. 12, 677-681], but reveals a considerable improvement in precision. The standard uncertainties of the room-temperature structure determination are about seven times lower than those of the neutron diffraction structure determination and about 13 times lower at $100 \mathrm{~K}$, due to the decrease in the amplitude of librations. The crystal expansion could be approximated linearly with a thermal volumic expansion coefficient of $1.19(12) \times 10^{-4} \mathrm{~K}^{-1}$. $\mathrm{NaTcO}_{4}$ adopts the scheelite $\left(\mathrm{CaWO}_{4}\right)$ structure type in space group type $I 4_{1} / a$ with $\mathrm{Na}$ and $\mathrm{Tc}$ atoms (both with site symmetry $\overline{4}$ ) replacing $\mathrm{Ca}$ and $\mathrm{W}$ atoms, respectively.

\section{Chemical context}

Sodium pertechnetate, $\mathrm{NaTcO}_{4}$, refers to a group of $d^{0}$-tetroxide anion salts. Since the inception of quantum chemistry, compounds of this type have been models (generally with respect to the $\mathrm{MnO}_{4}{ }^{-}$anion) for which the validity of the proposed equations and approximations for the case of $d$ electrons are verified. It was believed that, owing to the $d^{0}$ electronic state, they define the least complex class of compounds of $d$-elements. Such simplicity, due to the absence of $d$-electrons and their pseudospherical symmetry, does by far not imply that any of these compounds show no complex behavior under changing environmental conditions, e.g. by changing temperature and/or the strength of the crystal field, and publications on the discovery of a more complex behaviour and properties appeared periodically. For example, for sodium (German et al., 1987b, 1993), potassium (German et al., 1993; Gafurov \& Aliev, 2005) and caesium (Tarasov et al., 1991, 1992) tetraoxidotechnates, the existence of phase transitions was noted at high temperatures, while for the rhenium analogue, caesium tetraoxidorhenate, the ability of laserexcited second harmonic generation has been observed (Stefanovich et al., 1991). Differences for these systems are also observed in the crystal structures. Potassium permanganate crystallizes in the orthorhombic system (Palenik, 1967), whereas the pertechnetate and perrhenate of the same cation 
crystallize in the tetragonal system (Hoppe et al., 1999; Schwochau, 1962). Next to the interest for the $\mathrm{TcO}_{4}{ }^{-}$anion in its sodium salt, sodium cations in general are worth being investigated in detail. For example, sodium salts are known to form hydrates with different hydration numbers and various coordination numbers for the sodium cation. The change in these numbers often occurs in the vitally important temperature range of 309-313 K (German et al., 1987b; Tarasov et al., 2015). Precise structural data of such systems are important for the analyses of transmutation rates in homogeneous systems as noted by Kuo et al. (2017) and in this respect, are more useful than the data of previously determined structures (Kuo et al., 2017; Ackerman et al., 2016; German et al., 1987a; Spitsyn et al., 1987; Tarasov et al., 1983, 1991). Likewise, Ackerman et al. (2016) have shown that precise structural data are needed for the estimation of the incorporation possibility

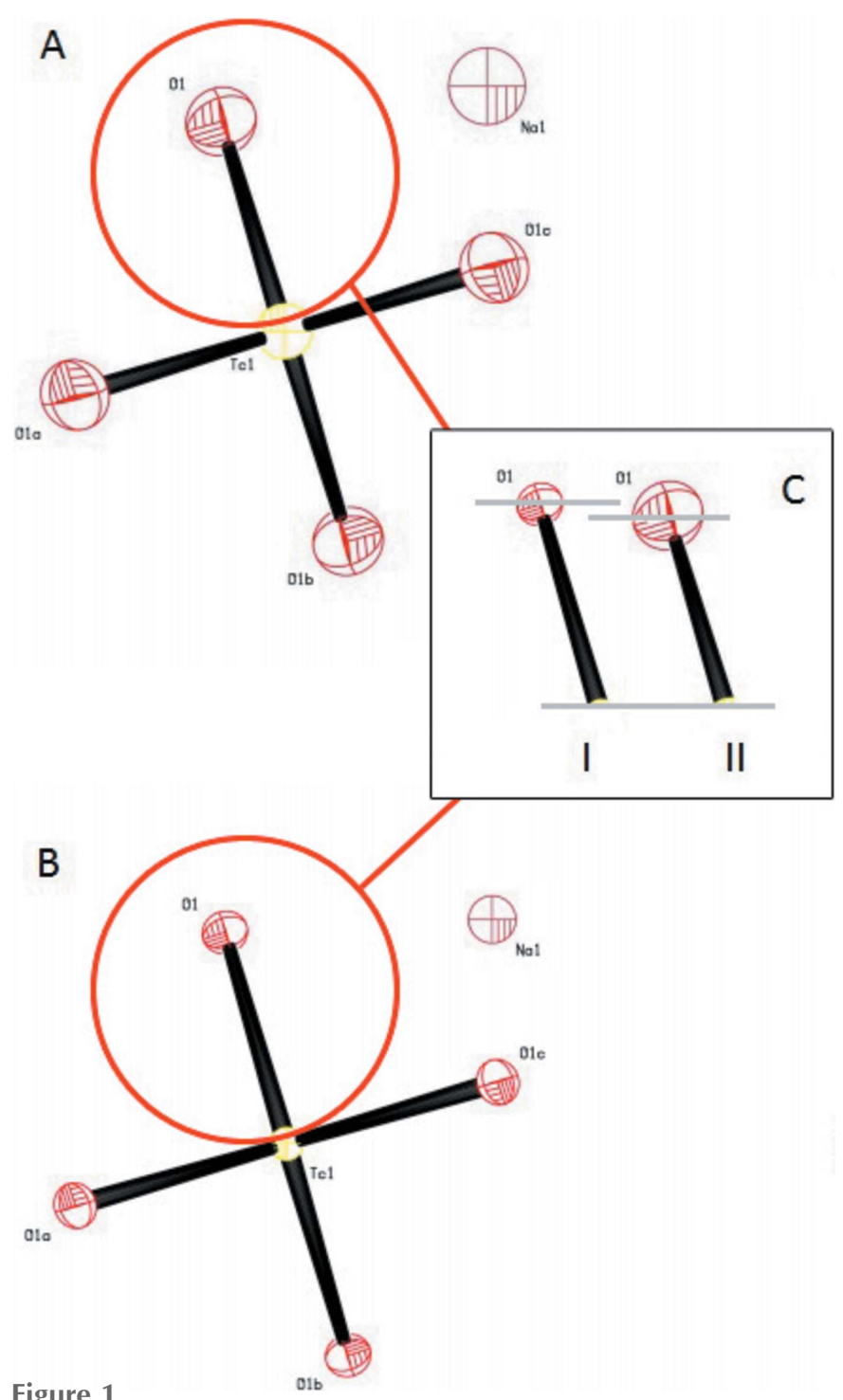

Figure 1

The elongation of bonds upon cooling from $296 \mathrm{~K}$ [(II), A] to $100 \mathrm{~K}[(\mathrm{I})$, B] is associated with a decrease in libration (C). All displacement ellipsoids are drawn at the $50 \%$ probability. [Symmetry codes: $(a)-y+\frac{5}{4}$, $x+\frac{1}{4},-z+\frac{1}{4} ;(b)-x+1,-y+\frac{3}{2}, z ;(c) y+\frac{3}{2},-x+\frac{11}{4}, z+\frac{3}{4}$.]
Table 1

Selected geometric parameters $\left(\AA,^{\circ}\right)$ at $100 \mathrm{~K}$.

\begin{tabular}{lrlr}
\hline $\mathrm{Tc} 1-\mathrm{O} 1$ & $1.7208(3)$ & $\mathrm{Na} 1-\mathrm{O} 1^{\mathrm{ii}}$ & $2.5980(4)$ \\
$\mathrm{Na} 1-\mathrm{O} 1^{\mathrm{i}}$ & $2.5107(4)$ & & \\
$\mathrm{O}^{\mathrm{iii}}-\mathrm{Tc} 1-\mathrm{O} 1$ & $108.439(12)$ & $\mathrm{O}^{\mathrm{iv}}-\mathrm{Tc} 1-\mathrm{O} 1$ & $111.56(3)$ \\
\hline
\end{tabular}

Symmetry codes: (i) $-x+1,-y+1,-z$; (ii) $x-1, y, z$; (iii) $-y+\frac{5}{4}, x+\frac{1}{4},-z+\frac{1}{4}$; (iv) $-x+1,-y+\frac{3}{2}, z$.

Table 2

Selected geometric parameters $\left(\AA{ }^{\circ}{ }^{\circ}\right)$ at $296 \mathrm{~K}$.

\begin{tabular}{lrlr}
\hline $\mathrm{Tc} 1-\mathrm{O} 1$ & $1.7183(6)$ & $\mathrm{O} 1-\mathrm{Na} 1^{\mathrm{ii}}$ & $2.6304(6)$ \\
$\mathrm{O} 1-\mathrm{Na} 1^{\mathrm{i}}$ & $2.5357(6)$ & & \\
$\mathrm{O} 1-\mathrm{Tc} 1-\mathrm{O} 1^{\mathrm{iii}}$ & $111.53(5)$ & $\mathrm{O} 1-\mathrm{Tc} 1-\mathrm{O} 1^{\mathrm{iv}}$ & $108.45(2)$ \\
\hline
\end{tabular}

Symmetry codes: (i) $-x+1,-y+1,-z$; (ii) $x+1, y, z$; (iii) $-x+1,-y+\frac{3}{2}, z$; (iv) $y-\frac{1}{4},-x+\frac{5}{4},-z+\frac{1}{4}$.

for ${ }^{99} \mathrm{Tc}$ into stable scheelite matrices of different compositions. Another aspect for obtaining more precise structure data on pertechnates is to clarify if pseudo-Jahn-Teller distortions of $d^{0}$-tetraoxide anions really take place when compared with previous determinations (German et al., 1987a; Spitsyn et al., 1987; Tarasov et al., 1983, 1991). In this context we have reinvestigated the crystal structure of $\mathrm{NaTcO}_{4}$ that is known from powder diffraction data only, namely by inspection of its X-ray powder diffraction pattern (Schwochau, 1962; Keller \& Kanellakopulos, 1963) and Rietveld refinement of neutron powder diffraction data (Weaver et al., 2017).

\section{Structural commentary}

The structure of anhydrous $\mathrm{NaTcO}_{4}$, determined here on the basis of X-ray diffraction data of a single crystal recorded both at room and low temperature, belongs to the $\mathrm{CaWO}_{4}$ structural type (space group type $I 4_{1} / a$ ). The obtained bond lengths and angles are similar to those obtained from previous X-ray powder (Keller \& Kanellakopulos, 1963; Schwochau, 1962) and neutron powder diffraction studies (Weaver et al., 2017)

Lattice parameters determined here with the precision of $0.0002-0.0005 \AA$ at $296 \mathrm{~K}$ (Table 1) are close to those of $a=$

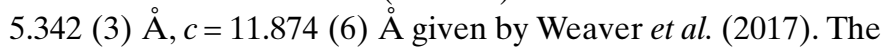
lattice parameters at $100 \mathrm{~K}$ are $a=5.2945$ (2) $\AA, c=$ 11.7470 (5) $\AA$ (single crystal measurement). These values represent the thermal volumic expansion coefficient of $1.19(12) \times 10^{-4} \mathrm{~K}^{-1}$. The $c / a$ ratio in this structure changes from 2.2187 (7) to 2.2223 (4) as a function of the temperature change from 100 to $296 \mathrm{~K}$.

Our results confirm that $\mathrm{NaTcO}_{4}$ is isostructural to $\mathrm{KTcO}_{4}$ and $\mathrm{RbTcO}_{4}$ (Keller \& Kanellakopulos, 1963). The structure is composed of three atom types $(\mathrm{Na}, \mathrm{Tc}, \mathrm{O})$. The $\mathrm{Tc}$ and $\mathrm{Na}$ atoms occupy special positions with $\overline{4}$ symmetry, Wyckoff positions $4 b$ and $4 a$, respectively. The configuration of the $\mathrm{TeO}_{4}{ }^{-}$anion is that of a slightly distorted tetrahedron both at $296 \mathrm{~K}$ and at $100 \mathrm{~K}$ (Tables 1 and 2). The Tc-O distances are 1.7183 (6) $\AA$ at $296 \mathrm{~K}$ and 1.7208 (3) $\AA$ at $100 \mathrm{~K}$. These distances are in good agreement with values known for these ions from the literature (German et al., 1987a; Tarasov et al., 


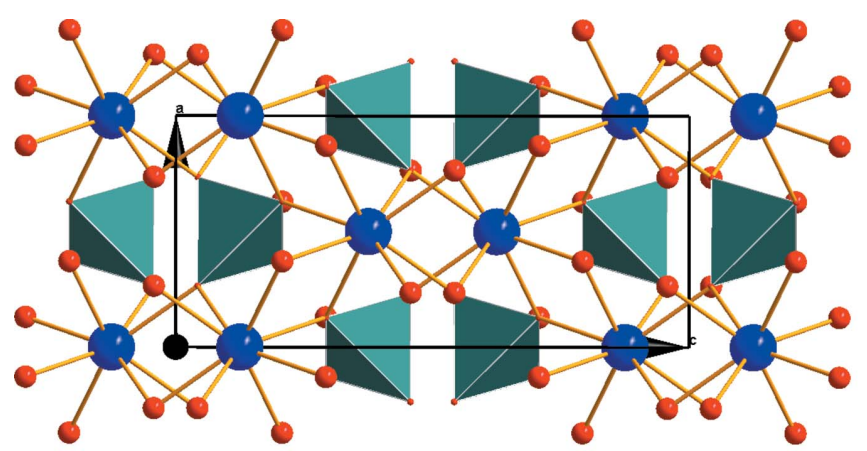

Figure 2

View of the crystal packing of the title compound.

1992; Kuo et al., 2017; Ackerman et al., 2016). The elongation of bonds (Fig. 1), while decreasing the temperature from $296 \mathrm{~K}$ to $100 \mathrm{~K}$, can be attributed to a decrease in the libration effect (German et al., 1987a). A similar phenomenon has previously been observed in the structure of anilinium pertechnetate (Maruk et al., 2010).

The greatest distortion of the $\mathrm{TcO}_{4}{ }^{-}$anion from an ideal tetrahedral configuration reported by Weaver et al. (2017) is confirmed by our analysis of the $\mathrm{O}-\mathrm{Tc}-\mathrm{O}$ angles in the $\mathrm{NaTcO}_{4}$ structure, but the difference is not as high as in the model from the neutron diffraction experiment (Weaver et al., 2017). The maximum deviation values are $3.12^{\circ}$ at $100 \mathrm{~K}$ and $3.08^{\circ}$ at $296 \mathrm{~K}$ for the sodium salt and are larger in comparison with the potassium and rubidium salts, because the sodium cation has the smallest ionic radius compared to $\mathrm{K}^{+}$and $\mathrm{Rb}^{+}$

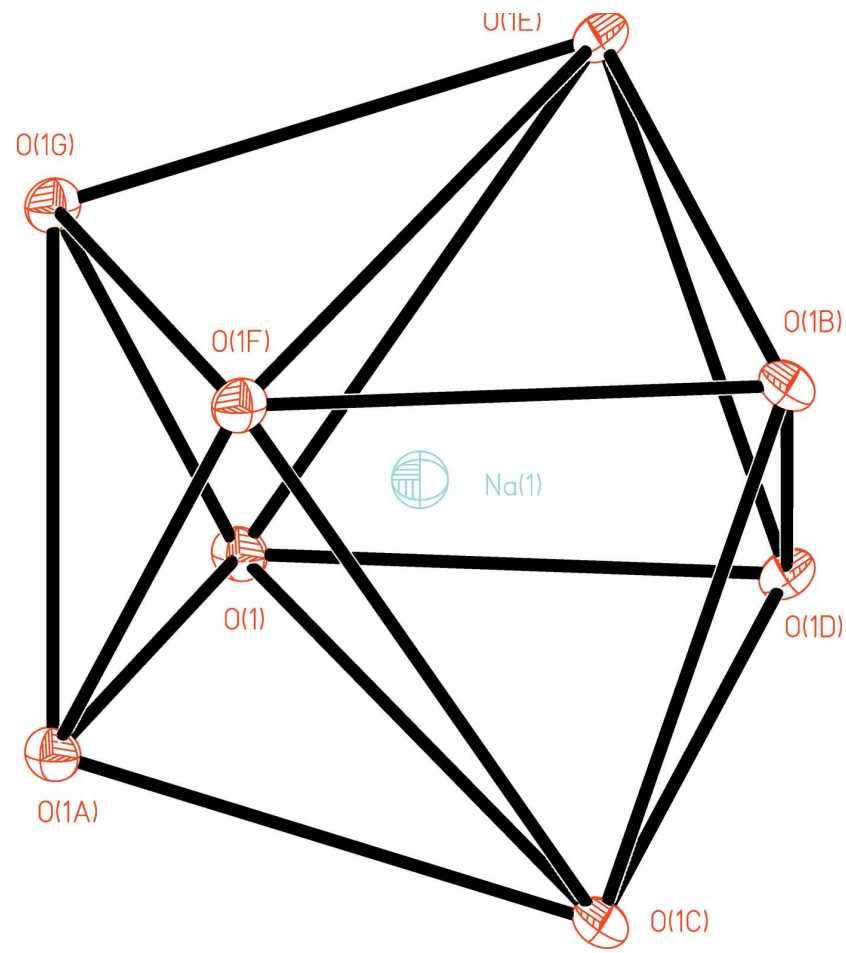

Figure 3

The coordination polyhedron of the sodium cation (data from $100 \mathrm{~K}$ measurement).

and hence has the highest polarizing ability. This distortion is insensitive to the temperature change from $100 \mathrm{~K}$ to $296 \mathrm{~K}$.

The packing of $\mathrm{Na}^{+}$cations and $\mathrm{TcO}_{4}{ }^{-}$anions in the crystal is presented in Fig. 2. Each $\mathrm{Na}^{+}$cation is coordinated by eight

Table 3

Experimental details.

$100 \mathrm{~K}$

Crystal data

Chemical formula

$M_{\mathrm{r}}$

Crystal system, space group

Temperature (K)

$a, c(\AA)$

$V\left(\AA^{3}\right)$

Z

Radiation type

$\mu\left(\mathrm{mm}^{-1}\right)$

Crystal size (mm)

Data collection

Diffractometer

Absorption correction

$T_{\min }, T_{\max }$

No. of measured, independent and observed $[I>2 \sigma(I)]$ reflections

$R_{\text {int }}$

$(\sin \theta / \lambda)_{\max }\left(\AA^{-1}\right)$

Refinement

$R\left[F^{2}>2 \sigma\left(F^{2}\right)\right], w R\left(F^{2}\right), S$

No. of reflections

No. of parameters

$\Delta \rho_{\max }, \Delta \rho_{\min }\left(\mathrm{e}^{-3}\right)$
$\mathrm{NaTcO}_{4}$

185.9

Tetragonal, $I 4_{1} / a$

100

5.2945 (2), 11.7470 (5)

329.29 (3)

4

Mo $K \alpha$

4.33

$0.34 \times 0.28 \times 0.20$

Bruker Kappa APEXII area-detector Multi-scan (SADABS; Bruker, 2008) $0.399,0.478$

$6909,678,661$

0.018

0.995

$0.009,0.017,1.31$

678

15

$0.26,-0.34$
$296 \mathrm{~K}$

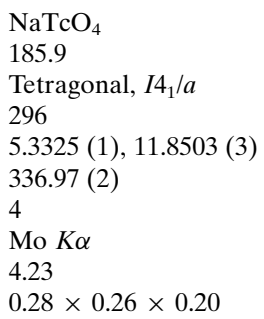

Bruker Kappa APEXII area-detector Multi-scan (SADABS; Bruker, 2008) $0.386,0.485$ $2371,365,350$

0.016

0.805

$0.008,0.019,1.16$

365

15

$0.23,-0.33$

Computer programs: APEX2 and SAINT-Plus (Bruker, 2008), SHELXS2014 (Sheldrick, 2015a), SHELXL2014 (Sheldrick, 2015b) and SHELXTL (Sheldrick, 2008). 
oxygen atoms that are belonging to four $\mathrm{TcO}_{4}{ }^{-}$anions. The resulting coordination polyhedron can be described as a distorted dodecahedron (Fig. 3). The two dihedral angles between pairs of two triangular faces sharing an edge that connects two five-edged vertices of the dodecahedron are equal to 21.2 and $30.3^{\circ}$, respectively. The corresponding faces should form an angle of $29.5^{\circ}$ for a dodecahedron and $0^{\circ}$ for a square anti-prism according to the Aslanov-Porai-Koshits criterion (Porai-Koshits \& Aslanov, 1972). Hence the coordination polyhedron of the sodium cation is closer to a dodecahedron than to a square anti-prism. Each of the four oxygen atoms of an individual $\mathrm{TcO}_{4}^{-}$anion is in contact with two sodium cations, so that each $\mathrm{TcO}_{4}{ }^{-}$anion is directly contacted with eight sodium cations.

\section{Synthesis and crystallization}

The synthesis of the title compound was carried out based on neutralization of an aqueous solution of freshly prepared $\mathrm{HTcO}_{4}$ with an equivalent quantity of $1 \mathrm{M}$ aqueous solution of chemically pure sodium hydroxide. The $\mathrm{HTcO}_{4}$ solution was made by dissolution of $\mathrm{Tc}_{2} \mathrm{O}_{7}$ sublimed from $\mathrm{TcO}_{2}$ in an oxygen flow at $973 \mathrm{~K}$.

\section{Refinement}

Crystal data, data collection and structure refinement details are summarized in Table 3. Seven (six) reflections at room (and low) temperature were omitted from refinement due to large differences between observed and calculated intensities.

\section{References}

Ackerman, M., Kim, E., Weck, P. F., Chernesky, W. \& Czerwinski, K. R. (2016). Dalton Trans. 45, 18171-18176.
Bruker (2008). APEX2, SAINT-Plus and SADABS. Bruker AXS Inc., Madison, Wisconsin, USA.

Gafurov, M. M. \& Aliev, A. R. (2005). J. Struct. Chem. 46, 824828.

German, K. E., Grigoriev, M. S. \& Kuzina, A. (1987a). Zh. Neorg. Khim. 32, 1089-1095.

German, K. E., Grushevschkaya, L. N., Kryutchkov, S. V., Pustovalov, V. A. \& Obruchikov, V. V. (1993). Radiochim. Acta, 63, 221-224.

German, K. E., Kryuchkov \& S. V., Belyaeva, L. I. (1987b). Izv. Akad. Nauk SSSR Ser. Khim. 10, 2387.

Hoppe, R., Fischer, D. \& Schneider, J. (1999). Z. Anorg. Allg. Chem. 625, 1135-1142.

Keller, C. \& Kanellakopulos, B. (1963). Radiochim. Acta, 1, 107108.

Kuo, E. Y., Qin, M. J., Thorogood, G. J., Huai, P., Ren, C. L., Lumpkin, G. R. \& Middleburgh, S. C. (2017). Modell. Simul. Mater. Sci. Eng. 25, 025011.

Maruk, A. Ya., Grigor'ev, M. S. \& German, K. E. (2010). Russ. J. Coord. Chem. 36, 381-388.

Palenik, G. J. (1967). Inorg. Chem. 6, 504-507.

Porai-Koshits, M. A. \& Aslanov, L. A. (1972). Russ. J. Struct. Chem. 12, 266.

Schwochau, K. (1962). Z. Naturforsch. Teil A, 17, 630.

Sheldrick, G. M. (2008). Acta Cryst. A64, 112-122.

Sheldrick, G. M. (2015a). Acta Cryst. A71, 3-8.

Sheldrick, G. M. (2015b). Acta Cryst. C71, 3-8.

Spitsyn, V. I., Kuzina, A. F., German, K. E. \& Grigor'ev, M. S. (1987). Dokl. Akad. Nauk SSSR, 293, 101-104.

Stefanovich, S. Y., Kalinin, V. B., German, K. E. \& Elvaer, S. M. (1991). Zh. Neorg. Khim. 36, 2200-2202.

Tarasov, V. P., Kirakosyan, G. A. \& German, K. E. (1992). Z. Naturforsch. Teil A, 47, 325-329.

Tarasov, V. P., Kirakosyan, G. A. \& German, K. E. (2015). Russ. J. Phys. Chem. B, 9, 185-192.

Tarasov, V. P., Kirakosyan, G. A., German, K. E. \& Grigoriev, M. S. (1991). Russ. J. Coord. Chem. 17, 1643-1653.

Tarasov, V. P., Privalov, V. I., Petrushin, S. A., Kirakosian, G. A. \& Kriuchkov, S. V. (1983). Dokl. Akad. Nauk SSSR, 272, 919-920.

Weaver, J., Soderquist, C. Z., Washton, N. M., Lipton, A. S., Gassman, P. L., Lukens, W. W., Kruger, A. A., Wall, N. A. \& McCloy, J. S. (2017). Inorg. Chem. 56, 2533-2544. 


\section{supporting information}

Acta Cryst. (2017). E73, 1037-1040 [https://doi.org/10.1107/S2056989017008362]

\section{Redetermination of the crystal structure of $\mathrm{NaTcO}_{4}$ at 100 and $296 \mathrm{~K}$ based on single-crystal X-ray data}

Konstantin E. German, Mikhail S. Grigoriev, Bogdan L. Garashchenko, Alexander V. Kopytin and

\section{Ekaterina A. Tyupina}

\section{Computing details}

For both compounds, data collection: APEX2 (Bruker, 2008); cell refinement: SAINT-Plus (Bruker, 2008); data reduction: SAINT-Plus (Bruker, 2008); program(s) used to solve structure: SHELXS2014 (Sheldrick, 2015a); program(s) used to refine structure: SHELXL2014 (Sheldrick, 2015b); molecular graphics: SHELXTL (Sheldrick, 2008); software used to prepare material for publication: SHELXTL (Sheldrick, 2008).

(I) Sodium pertechnetate

Crystal data

$\mathrm{NaTcO}_{4}$

$$
M_{r}=185.9
$$

Tetragonal, $I 4_{1} / a$

$a=5.2945(2) \AA$

$c=11.7470(5) \AA$

$V=329.29(3) \AA^{3}$

$Z=4$

$F(000)=344$

$D_{\mathrm{x}}=3.750 \mathrm{Mg} \mathrm{m}^{-3}$

Data collection

Bruker Kappa APEXII area-detector diffractometer

$\omega$ - and $\varphi$-scans

Absorption correction: multi-scan

(SADABS; Bruker, 2008)

$T_{\min }=0.399, T_{\max }=0.478$

6909 measured reflections

Refinement

Refinement on $F^{2}$

Least-squares matrix: full

$R\left[F^{2}>2 \sigma\left(F^{2}\right)\right]=0.009$

$w R\left(F^{2}\right)=0.017$

$S=1.31$

678 reflections

15 parameters

0 restraints
Melting point $<1063 \mathrm{~K}$

Mo $K \alpha$ radiation, $\lambda=0.71073 \AA$

Cell parameters from 5622 reflections

$\theta=4.2-45.4^{\circ}$

$\mu=4.33 \mathrm{~mm}^{-1}$

$T=100 \mathrm{~K}$

Fragment, colourless

$0.34 \times 0.28 \times 0.20 \mathrm{~mm}$

678 independent reflections

661 reflections with $I>2 \sigma(I)$

$R_{\text {int }}=0.018$

$\theta_{\text {max }}=45.0^{\circ}, \theta_{\min }=4.2^{\circ}$

$h=-10 \rightarrow 10$

$k=-10 \rightarrow 10$

$l=-22 \rightarrow 23$ 
Fractional atomic coordinates and isotropic or equivalent isotropic displacement parameters $\left(\AA^{2}\right)$

\begin{tabular}{lllll}
\hline & $x$ & $y$ & $z$ & $U_{\text {iso }} * U_{\text {eq }}$ \\
\hline Tc1 & 0.5000 & 0.7500 & 0.1250 & $0.00517(2)$ \\
Na1 & 0.0000 & 0.2500 & 0.1250 & $0.00980(7)$ \\
O1 & $0.73565(6)$ & $0.62081(7)$ & $0.04262(3)$ & $0.00879(5)$ \\
\hline
\end{tabular}

Atomic displacement parameters $\left(\AA^{2}\right)$

\begin{tabular}{lllllll}
\hline & $U^{11}$ & $U^{22}$ & $U^{33}$ & $U^{12}$ & $U^{13}$ & $U^{23}$ \\
\hline Tc1 & $0.00489(2)$ & $0.00489(2)$ & $0.00574(3)$ & 0.000 & 0.000 & 0.000 \\
Na1 & $0.00988(10)$ & $0.00988(10)$ & $0.00965(16)$ & 0.000 & 0.000 & 0.000 \\
O1 & $0.00795(11)$ & $0.00901(11)$ & $0.00942(11)$ & $0.00054(9)$ & $0.00202(10)$ & $-0.00104(10)$ \\
\hline
\end{tabular}

Geometric parameters $\left(\AA,{ }^{\circ}\right)$

\begin{tabular}{|c|c|c|c|}
\hline $\mathrm{Tc} 1-\mathrm{O} 1^{\mathrm{i}}$ & $1.7208(3)$ & $\mathrm{Na} 1-\mathrm{O} 1^{\text {viii }}$ & $2.5980(4)$ \\
\hline $\mathrm{Tc} 1-\mathrm{O} 1^{\mathrm{ii}}$ & $1.7208(3)$ & $\mathrm{Na} 1-\mathrm{O} 1^{\text {ix }}$ & $2.5980(4)$ \\
\hline $\mathrm{Tc} 1-\mathrm{O} 1^{\mathrm{iii}}$ & $1.7208(3)$ & $\mathrm{Na} 1-\mathrm{O}^{\mathrm{x}}$ & $2.5980(4)$ \\
\hline $\mathrm{Tc} 1-\mathrm{O} 1$ & $1.7208(3)$ & $\mathrm{Na} 1-\mathrm{Na} 1^{\mathrm{xi}}$ & $3.9538(1)$ \\
\hline $\mathrm{Na} 1-\mathrm{O} 1^{\text {iv }}$ & $2.5107(4)$ & $\mathrm{Na} 1-\mathrm{Na} 1^{\mathrm{xii}}$ & $3.9538(1)$ \\
\hline $\mathrm{Na} 1-\mathrm{O} 1^{\mathrm{v}}$ & $2.5107(4)$ & $\mathrm{Na} 1-\mathrm{Na} 1^{\mathrm{xiii}}$ & $3.9538(1)$ \\
\hline $\mathrm{Na} 1-\mathrm{O}^{\mathrm{vi}}$ & $2.5107(4)$ & $\mathrm{Na} 1-\mathrm{Na}^{\mathrm{xiv}}$ & $3.9538(1)$ \\
\hline $\mathrm{Na} 1-\mathrm{O} 1^{\mathrm{vii}}$ & $2.5107(4)$ & $\mathrm{O} 1-\mathrm{Na} 1^{\mathrm{vii}}$ & $2.5107(4)$ \\
\hline $\mathrm{Na} 1-\mathrm{O} 1^{\mathrm{iii}}$ & $2.5980(4)$ & $\mathrm{O} 1-\mathrm{Na} 1^{\mathrm{xv}}$ & $2.5980(4)$ \\
\hline $\mathrm{O} 1^{\mathrm{i}-\mathrm{Tc}} 1-\mathrm{O} 1^{\mathrm{ii}}$ & $108.439(12)$ & $\mathrm{O} 1^{\mathrm{iii}}-\mathrm{Na} 1-\mathrm{Na} 1^{\mathrm{xi}}$ & $129.596(8)$ \\
\hline 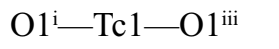 & $111.56(3)$ & $\mathrm{O} 1^{\mathrm{vii}}-\mathrm{Na} 1-\mathrm{Na} 1^{\mathrm{xi}}$ & $85.180(8)$ \\
\hline $\mathrm{O} 1^{\mathrm{ii}-\mathrm{Tc}} 1-\mathrm{O} 1^{\mathrm{iii}}$ & $108.439(12)$ & $\mathrm{O} 1^{\mathrm{ix}}-\mathrm{Na} 1-\mathrm{Na} 1^{\mathrm{xi}}$ & $38.498(8)$ \\
\hline $\mathrm{O} 1{ }^{\mathrm{i}}-\mathrm{Tc} 1-\mathrm{O} 1$ & $108.439(12)$ & $\mathrm{O} 1^{\mathrm{x}}-\mathrm{Na} 1-\mathrm{Na} 1^{\mathrm{xi}}$ & $103.255(8)$ \\
\hline $\mathrm{O} 1 \mathrm{ii}-\mathrm{Tc} 1-\mathrm{O} 1$ & $111.56(3)$ & $\mathrm{O} 1^{\mathrm{iv}}-\mathrm{Na} 1-\mathrm{Na} 1^{\mathrm{xii}}$ & $162.891(8)$ \\
\hline $\mathrm{O} 1{ }^{\mathrm{iii}}-\mathrm{Tc} 1-\mathrm{O} 1$ & $108.439(12)$ & $\mathrm{O} 1^{\mathrm{v}}-\mathrm{Na} 1-\mathrm{Na} 1^{\mathrm{xii}}$ & $66.415(8)$ \\
\hline $\mathrm{O} 1^{\mathrm{iv}}-\mathrm{Na} 1-\mathrm{O} 1^{\mathrm{v}}$ & $127.954(10)$ & 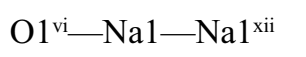 & $40.101(8)$ \\
\hline $\mathrm{O} 1^{\mathrm{iv}}-\mathrm{Na} 1-\mathrm{O}^{\mathrm{vi}}$ & $127.954(10)$ & $\mathrm{O} 1^{\mathrm{vii}}-\mathrm{Na} 1-\mathrm{Na} 1^{\mathrm{xii}}$ & $102.079(9)$ \\
\hline $\mathrm{O} 1^{\mathrm{v}}-\mathrm{Na} 1-\mathrm{O} 1^{\mathrm{vi}}$ & $76.700(17)$ & $\mathrm{O} 1^{\mathrm{iii}}-\mathrm{Na} 1-\mathrm{Na} 1^{\mathrm{xii}}$ & $38.498(8)$ \\
\hline $\mathrm{O} 1^{\mathrm{iv}}-\mathrm{Na} 1-\mathrm{O} 1^{\mathrm{vii}}$ & $76.700(17)$ & $\mathrm{O} 1^{\mathrm{vii}}-\mathrm{Na} 1-\mathrm{Na} 1^{\mathrm{xii}}$ & $103.255(8)$ \\
\hline $\mathrm{O} 1^{\mathrm{v}}-\mathrm{Na} 1-\mathrm{O}^{\mathrm{vii}}$ & $127.954(10)$ & $\mathrm{O} 1^{\mathrm{ix}}-\mathrm{Na} 1-\mathrm{Na} 1^{\mathrm{xii}}$ & $85.180(8)$ \\
\hline $\mathrm{O} 1^{\mathrm{vi}}-\mathrm{Na} 1-\mathrm{O} 1^{\mathrm{vii}}$ & $127.954(10)$ & $\mathrm{O} 1^{\mathrm{x}}-\mathrm{Na} 1-\mathrm{Na} 1^{\mathrm{xii}}$ & $129.596(8)$ \\
\hline $\mathrm{O} 1^{\mathrm{iv}}-\mathrm{Na} 1-\mathrm{O} 1^{\mathrm{iii}}$ & $149.332(14)$ & $\mathrm{Na} 1^{\mathrm{xi}}-\mathrm{Na} 1-\mathrm{Na} 1^{\mathrm{xii}}$ & $123.484(2)$ \\
\hline $\mathrm{O} 1^{\mathrm{v}}-\mathrm{Na} 1-\mathrm{O} 1^{\mathrm{iii}}$ & $67.259(8)$ & $\mathrm{O} 1^{\mathrm{iv}}-\mathrm{Na} 1-\mathrm{Na} 1^{\mathrm{xiii}}$ & $66.415(8)$ \\
\hline 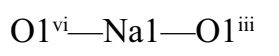 & $78.599(12)$ & $\mathrm{O} 1^{\mathrm{v}}-\mathrm{Na} 1-\mathrm{Na} 1^{\mathrm{xiii}}$ & $102.079(9)$ \\
\hline $\mathrm{O} 1^{\mathrm{vii}}-\mathrm{Na} 1-\mathrm{O} 1^{\mathrm{iii}}$ & $73.985(7)$ & $\mathrm{O} 1^{\mathrm{vi}}-\mathrm{Na} 1-\mathrm{Na} 1^{\mathrm{xiii}}$ & $162.891(8)$ \\
\hline $\mathrm{O} 1^{\mathrm{iv}}-\mathrm{Na} 1-\mathrm{O} 1^{\text {viii }}$ & $73.985(7)$ & $\mathrm{O} 1^{\mathrm{vii}}-\mathrm{Na} 1-\mathrm{Na} 1^{\mathrm{xiii}}$ & $40.101(8)$ \\
\hline $\mathrm{O} 1^{\mathrm{v}}-\mathrm{Na} 1-\mathrm{O} 1^{\text {viii }}$ & $78.599(12)$ & $\mathrm{O} 1^{\mathrm{iii}}-\mathrm{Na} 1-\mathrm{Na} 1^{\mathrm{xiii}}$ & $85.180(8)$ \\
\hline 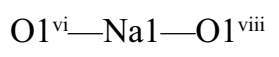 & $67.259(8)$ & 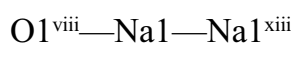 & $129.596(8)$ \\
\hline $\mathrm{O} 1^{\mathrm{vii}}-\mathrm{Na} 1-\mathrm{O} 1^{\mathrm{vii}}$ & $149.332(14)$ & $\mathrm{O} 1^{\mathrm{ix}}-\mathrm{Na} 1-\mathrm{Na} 1^{\mathrm{xiii}}$ & $103.255(8)$ \\
\hline $\mathrm{O} 1^{\mathrm{iii}}-\mathrm{Na} 1-\mathrm{O} 1^{\mathrm{viii}}$ & $136.261(16)$ & $\mathrm{O} 1^{\mathrm{x}}-\mathrm{Na} 1-\mathrm{Na} 1^{\mathrm{xiii}}$ & $38.498(8)$ \\
\hline $\mathrm{O} 1^{\mathrm{iv}}-\mathrm{Na} 1-\mathrm{O} 1^{\text {ix }}$ & $78.599(12)$ & $\mathrm{Na} 1^{\mathrm{xi}}-\mathrm{Na} 1-\mathrm{Na} 1^{\mathrm{xiii}}$ & $84.064(3)$ \\
\hline
\end{tabular}




\begin{tabular}{|c|c|c|c|}
\hline $\mathrm{O} 1^{\mathrm{v}}-\mathrm{Na} 1-\mathrm{O} 1^{\mathrm{ix}}$ & $149.332(14)$ & $\mathrm{Na} 1^{\mathrm{xii}}-\mathrm{Na} 1-\mathrm{Na} 1^{\mathrm{xiii}}$ & $123.484(2)$ \\
\hline 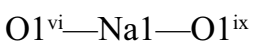 & $73.985(7)$ & $\mathrm{O} 1^{\mathrm{iv}}-\mathrm{Na} 1-\mathrm{Na} 1^{\text {xiv }}$ & $102.079(9)$ \\
\hline $\mathrm{O} 1^{\mathrm{vii}}-\mathrm{Na} 1-\mathrm{O} 1^{\mathrm{ix}}$ & $67.259(8)$ & $\mathrm{O} 1^{\mathrm{v}}-\mathrm{Na} 1-\mathrm{Na} 1^{\mathrm{xiv}}$ & $40.101(8)$ \\
\hline 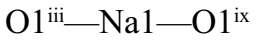 & $97.976(6)$ & $\mathrm{O} 1^{\mathrm{vi}}-\mathrm{Na} 1-\mathrm{Na} 1^{\text {xiv }}$ & $66.415(8)$ \\
\hline $\mathrm{O} 1^{\mathrm{viii}}-\mathrm{Na} 1-\mathrm{O} 1^{\mathrm{ix}}$ & $97.976(6)$ & $\mathrm{O} 1^{\mathrm{vii}}-\mathrm{Na} 1-\mathrm{Na} 1^{\mathrm{xiv}}$ & $162.891(8)$ \\
\hline $\mathrm{O} 1^{\mathrm{iv}}-\mathrm{Na} 1-\mathrm{O} 1^{\mathrm{x}}$ & $67.259(8)$ & $\mathrm{O} 1^{\mathrm{iii}}-\mathrm{Na} 1-\mathrm{Na} 1^{\mathrm{xiv}}$ & $103.255(8)$ \\
\hline $\mathrm{O} 1^{\mathrm{v}}-\mathrm{Na} 1-\mathrm{O}^{\mathrm{x}}$ & 73.985 (7) & $\mathrm{O} 1^{\mathrm{viii}}-\mathrm{Na} 1-\mathrm{Na} 1^{\mathrm{xiv}}$ & $38.498(8)$ \\
\hline $\mathrm{O} 1^{\mathrm{vi}}-\mathrm{Na} 1-\mathrm{O} 1^{\mathrm{x}}$ & $149.332(14)$ & $\mathrm{O} 1^{\mathrm{ix}}-\mathrm{Na} 1-\mathrm{Na} 1^{\mathrm{xiv}}$ & $129.596(8)$ \\
\hline 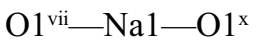 & $78.599(12)$ & $\mathrm{O} 1^{\mathrm{x}}-\mathrm{Na} 1-\mathrm{Na} 1^{\mathrm{xiv}}$ & $85.180(8)$ \\
\hline 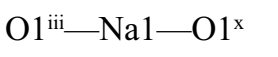 & $97.976(6)$ & $\mathrm{Na} 1^{\mathrm{xi}}-\mathrm{Na} 1-\mathrm{Na} 1^{\mathrm{xiv}}$ & $123.484(2)$ \\
\hline $\mathrm{O} 1^{\mathrm{vii}-\mathrm{Na} 1-\mathrm{O} 1^{\mathrm{x}}}$ & $97.976(6)$ & $\mathrm{Na} 1^{\mathrm{xii}}-\mathrm{Na} 1-\mathrm{Na} 1^{\mathrm{xiv}}$ & 84.064 (4) \\
\hline $\mathrm{O} 1^{\mathrm{ix}}-\mathrm{Na} 1-\mathrm{O} 1^{\mathrm{x}}$ & $136.261(16)$ & 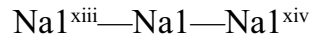 & $123.484(2)$ \\
\hline $\mathrm{O} 1^{\mathrm{iv}}-\mathrm{Na} 1-\mathrm{Na} 1^{\mathrm{xi}}$ & $40.101(8)$ & $\mathrm{Tc} 1-\mathrm{O} 1-\mathrm{Na} 1^{\mathrm{vii}}$ & $137.472(19)$ \\
\hline $\mathrm{O} 1^{\mathrm{v}}-\mathrm{Na} 1-\mathrm{Na} 1^{\mathrm{xi}}$ & $162.891(8)$ & $\mathrm{Tc} 1-\mathrm{O} 1-\mathrm{Na} 1^{\mathrm{xv}}$ & $118.781(18)$ \\
\hline $\mathrm{O} 1^{\mathrm{vi}}-\mathrm{Na} 1-\mathrm{Na} 1^{\mathrm{xi}}$ & $102.079(9)$ & $\mathrm{Na} 1^{\mathrm{vii}}-\mathrm{O} 1-\mathrm{Na} 1^{\mathrm{xv}}$ & $101.402(12)$ \\
\hline 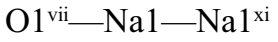 & $66.415(8)$ & & \\
\hline
\end{tabular}

Symmetry codes: (i) $-y+5 / 4, x+1 / 4,-z+1 / 4$; (ii) $-x+1,-y+3 / 2, z$; (iii) $y-1 / 4,-x+5 / 4,-z+1 / 4$; (iv) $x-1, y-1 / 2,-z$; (v) $y-3 / 4,-x+5 / 4, z+1 / 4$; (vi) $-y+3 / 4$, $x-3 / 4, z+1 / 4$; (vii) $-x+1,-y+1,-z$; (viii) $-y+1 / 4, x-3 / 4,-z+1 / 4$; (ix) $-x+1,-y+1 / 2, z$; (x) $x-1, y, z$; (xi) $-x,-y,-z$; (xii) $-x+1 / 2,-y+1 / 2,-z+1 / 2$; (xiii) $-x$, $-y+1,-z ;$ (xiv) $-x-1 / 2,-y+1 / 2,-z+1 / 2 ;$ (xv) $x+1, y, z$.

(II) Sodium tetraoxidotechnetate(VII)

Crystal data

\section{$\mathrm{O}_{4} \mathrm{Tc} \cdot \mathrm{Na}$}

$M_{r}=185.9$

Tetragonal, $I 4_{1} / a$

Hall symbol: -I 4ad

$a=5.3325(1) \AA$

$c=11.8503(3) \AA$

$V=336.97(2) \AA^{3}$

$Z=4$

$F(000)=344$

\section{Data collection}

Bruker Kappa APEX II area-detector diffractometer

Graphite monochromator

Detector resolution: 9.091 pixels $\mathrm{mm}^{-1}$

$\omega$ - and $\varphi$-scans

Absorption correction: multi-scan

(SADABS; Bruker, 2008)

$T_{\text {min }}=0.386, T_{\text {max }}=0.485$

\section{Refinement}

Refinement on $F^{2}$

Least-squares matrix: full

$R\left[F^{2}>2 \sigma\left(F^{2}\right)\right]=0.008$

$w R\left(F^{2}\right)=0.019$

$S=1.16$

365 reflections

15 parameters

0 restraints
$D_{\mathrm{x}}=3.664 \mathrm{Mg} \mathrm{m}^{-3}$

Melting point $<1063 \mathrm{~K}$

Mo $K \alpha$ radiation, $\lambda=0.71073 \AA$

Cell parameters from 2097 reflections

$\theta=4.2-35.2^{\circ}$

$\mu=4.23 \mathrm{~mm}^{-1}$

$T=296 \mathrm{~K}$

Fragment, colourless

$0.28 \times 0.26 \times 0.20 \mathrm{~mm}$

2371 measured reflections

365 independent reflections

350 reflections with $I>2 \sigma(I)$

$R_{\text {int }}=0.016$

$\theta_{\max }=34.9^{\circ}, \theta_{\min }=4.2^{\circ}$

$h=-8 \rightarrow 7$

$k=-8 \rightarrow 8$

$l=-18 \rightarrow 18$

$w=1 /\left[\sigma^{2}\left(F_{\mathrm{o}}^{2}\right)+(0.0071 P)^{2}+0.0846 P\right]$

where $P=\left(F_{\mathrm{o}}^{2}+2 F_{\mathrm{c}}{ }^{2}\right) / 3$

$(\Delta / \sigma)_{\max }<0.001$

$\Delta \rho_{\max }=0.23$ e $\AA^{-3}$

$\Delta \rho_{\min }=-0.33$ e $\AA^{-3}$

Extinction correction: SHELXL, $\mathrm{Fc}^{*}=\mathrm{kFc}\left[1+0.001 \mathrm{xFc}^{2} \lambda^{3} / \sin (2 \theta)\right]^{-1 / 4}$

Extinction coefficient: 0.136 (3) 
Fractional atomic coordinates and isotropic or equivalent isotropic displacement parameters $\left(\AA^{2}\right)$

\begin{tabular}{lllll}
\hline & $x$ & $y$ & $z$ & $U_{\text {iso }} * U_{\text {eq }}$ \\
\hline Tc1 & 0.5000 & 0.7500 & 0.1250 & $0.01434(6)$ \\
Na1 & 0.0000 & 0.2500 & 0.1250 & $0.02546(16)$ \\
O1 & $0.73494(12)$ & $0.62442(11)$ & $0.04342(6)$ & $0.02225(12)$ \\
\hline
\end{tabular}

Atomic displacement parameters $\left(\AA^{2}\right)$

\begin{tabular}{lllllll}
\hline & $U^{11}$ & $U^{22}$ & $U^{33}$ & $U^{12}$ & $U^{13}$ & $U^{23}$ \\
\hline Tc1 & $0.01355(6)$ & $0.01355(6)$ & $0.01591(7)$ & 0.000 & 0.000 & 0.000 \\
Na1 & $0.0261(2)$ & $0.0261(2)$ & $0.0242(4)$ & 0.000 & 0.000 & 0.000 \\
O1 & $0.0200(2)$ & $0.0225(2)$ & $0.0242(3)$ & $0.0005(2)$ & $0.0050(2)$ & $-0.0030(2)$ \\
\hline
\end{tabular}

Geometric parameters $\left(\AA,{ }^{\circ}\right)$

\begin{tabular}{|c|c|c|c|}
\hline $\mathrm{Tc} 1-\mathrm{O}^{\mathrm{i}}$ & $1.7183(6)$ & $\mathrm{Na} 1-\mathrm{O} 1^{\mathrm{vii}}$ & $2.5357(6)$ \\
\hline $\mathrm{Tc} 1-\mathrm{O} 1$ & $1.7183(6)$ & $\mathrm{Na} 1-\mathrm{O} 1^{\mathrm{iii}}$ & $2.6303(6)$ \\
\hline $\mathrm{Tc} 1-\mathrm{O} 1^{\mathrm{ii}}$ & $1.7183(6)$ & $\mathrm{Na} 1-\mathrm{O} 1^{\text {viii }}$ & $2.6303(6)$ \\
\hline $\mathrm{Tc} 1-\mathrm{O} 1^{\mathrm{iii}}$ & $1.7183(6)$ & $\mathrm{Na} 1-\mathrm{O} 1^{\mathrm{ix}}$ & $2.6303(6)$ \\
\hline $\mathrm{Na} 1-\mathrm{O} 1^{\text {iv }}$ & $2.5357(6)$ & $\mathrm{Na} 1-\mathrm{O} 1^{\mathrm{x}}$ & $2.6303(6)$ \\
\hline $\mathrm{Na} 1-\mathrm{O}^{\mathrm{v}}$ & $2.5357(6)$ & $\mathrm{O} 1-\mathrm{Na} 1^{\mathrm{vii}}$ & $2.5357(6)$ \\
\hline $\mathrm{Na} 1-\mathrm{O} 1^{\mathrm{vi}}$ & $2.5357(6)$ & $\mathrm{O} 1-\mathrm{Na} 1^{\mathrm{xi}}$ & $2.6304(6)$ \\
\hline $\mathrm{O} 1{ }^{\mathrm{i}}-\mathrm{Tc} 1-\mathrm{O} 1$ & $108.45(2)$ & $\mathrm{O} 1^{\mathrm{iii}}-\mathrm{Na} 1-\mathrm{Na} 1^{\mathrm{xii}}$ & $129.245(14)$ \\
\hline $\mathrm{O} 1^{\mathrm{i}}-\mathrm{Tc} 1-\mathrm{O} 1^{\mathrm{ii}}$ & $108.45(2)$ & $\mathrm{O} 1^{\mathrm{vii}}-\mathrm{Na} 1-\mathrm{Na} 1^{\mathrm{xii}}$ & $85.049(14)$ \\
\hline $\mathrm{O} 1-\mathrm{Tc} 1-\mathrm{O} 1^{\mathrm{ii}}$ & $111.53(5)$ & $\mathrm{O} 1^{\mathrm{ix}}-\mathrm{Na} 1-\mathrm{Na} 1^{\mathrm{xii}}$ & $38.652(13)$ \\
\hline 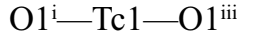 & $111.53(5)$ & $\mathrm{O} 1^{\mathrm{x}}-\mathrm{Na} 1-\mathrm{Na} 1^{\mathrm{xii}}$ & $103.569(14)$ \\
\hline $\mathrm{O} 1-\mathrm{Tc} 1-\mathrm{O} 1^{\mathrm{iii}}$ & $108.45(2)$ & $\mathrm{O} 1^{\mathrm{iv}}-\mathrm{Na} 1-\mathrm{Na} 1^{\text {xiii }}$ & $163.325(14)$ \\
\hline $\mathrm{O} 1^{\mathrm{ii}-\mathrm{Tc}} 1-\mathrm{O} 1^{\mathrm{iii}}$ & $108.45(2)$ & $\mathrm{O} 1^{\mathrm{v}}-\mathrm{Na} 1-\mathrm{Na} 1^{\mathrm{xiii}}$ & $65.896(14)$ \\
\hline $\mathrm{O} 1^{\mathrm{iv}}-\mathrm{Na} 1-\mathrm{O} 1^{\mathrm{v}}$ & $128.283(18)$ & $\mathrm{O} 1^{\mathrm{vi}}-\mathrm{Na} 1-\mathrm{Na} 1^{\mathrm{xiii}}$ & $40.383(14)$ \\
\hline $\mathrm{O}^{\mathrm{iv}}-\mathrm{Na} 1-\mathrm{O}^{\mathrm{vi}}$ & $128.283(18)$ & $\mathrm{O} 1^{\mathrm{vii}}-\mathrm{Na} 1-\mathrm{Na} 1^{\mathrm{xiii}}$ & $102.250(15)$ \\
\hline $\mathrm{O} 1^{\mathrm{v}}-\mathrm{Na} 1-\mathrm{O} 1^{\mathrm{vi}}$ & $76.17(3)$ & $\mathrm{O} 1^{\mathrm{iii}}-\mathrm{Na} 1-\mathrm{Na} 1^{\mathrm{xiii}}$ & $38.652(13)$ \\
\hline $\mathrm{O} 1^{\mathrm{iv}}-\mathrm{Na} 1-\mathrm{O} 1^{\mathrm{vii}}$ & $76.17(3)$ & $\mathrm{O} 1^{\mathrm{viii}}-\mathrm{Na} 1-\mathrm{Na} 1^{\text {xiii }}$ & $103.569(14)$ \\
\hline $\mathrm{O} 1^{\mathrm{v}}-\mathrm{Na} 1-\mathrm{O} 1^{\mathrm{vii}}$ & $128.283(18)$ & $\mathrm{O} 1^{\mathrm{ix}}-\mathrm{Na} 1-\mathrm{Na} 1^{\mathrm{xiii}}$ & $85.049(14)$ \\
\hline 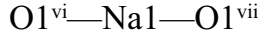 & $128.283(18)$ & $\mathrm{O} 1^{\mathrm{x}}-\mathrm{Na} 1-\mathrm{Na} 1^{\mathrm{xii}}$ & $129.245(14)$ \\
\hline $\mathrm{O} 1^{\mathrm{iv}}-\mathrm{Na} 1-\mathrm{O} 1^{\mathrm{iii}}$ & $148.68(2)$ & $\mathrm{Na} 1^{\mathrm{xii}}-\mathrm{Na} 1-\mathrm{Na} 1^{\text {xiii }}$ & $123.539(1)$ \\
\hline $\mathrm{O} 1^{\mathrm{v}}-\mathrm{Na} 1-\mathrm{O} 1^{\mathrm{iii}}$ & $67.149(15)$ & $\mathrm{O} 1^{\mathrm{iv}}-\mathrm{Na} 1-\mathrm{Na} 1^{\text {xiv }}$ & $65.896(14)$ \\
\hline 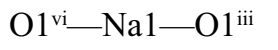 & $79.04(2)$ & $\mathrm{O} 1^{\mathrm{v}}-\mathrm{Na} 1-\mathrm{Na} 1^{\text {xiv }}$ & $102.250(15)$ \\
\hline 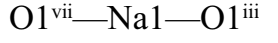 & $73.993(11)$ & $\mathrm{O} 1^{\mathrm{vi}}-\mathrm{Na} 1-\mathrm{Na} 1^{\mathrm{xiv}}$ & $163.325(14)$ \\
\hline $\mathrm{O} 1^{\mathrm{iv}}-\mathrm{Na} 1-\mathrm{O} 1^{\text {viii }}$ & $73.993(11)$ & $\mathrm{O} 1^{\mathrm{vii}}-\mathrm{Na} 1-\mathrm{Na} 1^{\mathrm{xiv}}$ & $40.383(14)$ \\
\hline $\mathrm{O} 1^{\mathrm{v}}-\mathrm{Na} 1-\mathrm{O} 1^{\text {viii }}$ & $79.04(2)$ & $\mathrm{O} 1^{\mathrm{iii}}-\mathrm{Na} 1-\mathrm{Na} 1^{\mathrm{xiv}}$ & $85.049(14)$ \\
\hline $\mathrm{O} 1^{\mathrm{vi}}-\mathrm{Na} 1-\mathrm{O} 1^{\mathrm{viii}}$ & $67.149(15)$ & $\mathrm{O} 1^{\mathrm{vii}}-\mathrm{Na} 1-\mathrm{Na} 1^{\mathrm{xiv}}$ & $129.245(14)$ \\
\hline 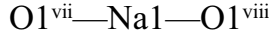 & $148.68(2)$ & $\mathrm{O} 1^{\mathrm{ix}}-\mathrm{Na} 1-\mathrm{Na} 1^{\mathrm{xiv}}$ & $103.569(14)$ \\
\hline 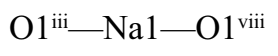 & $136.88(3)$ & $\mathrm{O} 1^{\mathrm{x}}-\mathrm{Na} 1-\mathrm{Na} 1^{\mathrm{xiv}}$ & $38.652(13)$ \\
\hline $\mathrm{O} 1^{\text {iv }}-\mathrm{Na} 1-\mathrm{O} 1^{\text {ix }}$ & $79.04(2)$ & $\mathrm{Na} 1^{\mathrm{xii}}-\mathrm{Na} 1-\mathrm{Na} 1^{\text {xiv }}$ & $83.973(2)$ \\
\hline $\mathrm{O} 1^{\mathrm{v}}-\mathrm{Na} 1-\mathrm{O} 1^{\mathrm{ix}}$ & $148.68(2)$ & 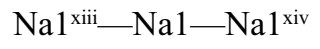 & $123.539(1)$ \\
\hline 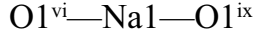 & $73.993(11)$ & $\mathrm{O} 1^{\mathrm{iv}}-\mathrm{Na} 1-\mathrm{Na} 1^{\mathrm{xv}}$ & $102.250(15)$ \\
\hline
\end{tabular}




\begin{tabular}{|c|c|c|c|}
\hline $\mathrm{O} 1^{\mathrm{vii}}-\mathrm{Na} 1-\mathrm{O} 1^{\mathrm{ix}}$ & $67.149(15)$ & $\mathrm{O} 1^{\mathrm{v}}-\mathrm{Na} 1-\mathrm{Na} 1^{\mathrm{xv}}$ & $40.383(14)$ \\
\hline 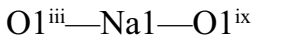 & $97.762(10)$ & $\mathrm{O} 1^{\mathrm{vi}}-\mathrm{Na} 1-\mathrm{Na} 1^{\mathrm{xv}}$ & $65.896(14)$ \\
\hline $\mathrm{O} 1^{\mathrm{vii}}-\mathrm{Na} 1-\mathrm{O} 1^{\mathrm{ix}}$ & $97.762(10)$ & $\mathrm{O} 1^{\mathrm{vii}}-\mathrm{Na} 1-\mathrm{Na} 1^{\mathrm{xv}}$ & $163.325(14)$ \\
\hline $\mathrm{O} 1^{\mathrm{iv}}-\mathrm{Na} 1-\mathrm{O} 1^{\mathrm{x}}$ & $67.149(15)$ & $\mathrm{O} 1^{\mathrm{iii}}-\mathrm{Na} 1-\mathrm{Na} 1^{\mathrm{xv}}$ & $103.569(14)$ \\
\hline $\mathrm{O} 1^{\mathrm{v}}-\mathrm{Na} 1-\mathrm{O}^{\mathrm{x}}$ & $73.993(11)$ & $\mathrm{O} 1^{\mathrm{viii}}-\mathrm{Na} 1-\mathrm{Na} 1^{\mathrm{xv}}$ & $38.652(13)$ \\
\hline 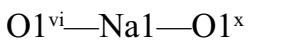 & $148.68(2)$ & $\mathrm{O} 1^{\mathrm{ix}}-\mathrm{Na} 1-\mathrm{Na} 1^{\mathrm{xv}}$ & $129.245(14)$ \\
\hline 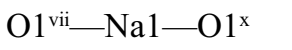 & $79.04(2)$ & $\mathrm{O} 1^{\mathrm{x}}-\mathrm{Na} 1-\mathrm{Na} 1^{\mathrm{xv}}$ & $85.049(14)$ \\
\hline $\mathrm{O} 1^{\mathrm{iii}}-\mathrm{Na} 1-\mathrm{O} 1^{\mathrm{x}}$ & $97.762(10)$ & $\mathrm{Na} 1^{\mathrm{xii}}-\mathrm{Na} 1-\mathrm{Na}^{\mathrm{xv}}$ & $123.539(1)$ \\
\hline 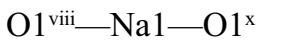 & $97.762(10)$ & $\mathrm{Na} 1^{\mathrm{xiii}}-\mathrm{Na} 1-\mathrm{Na} 1^{\mathrm{xv}}$ & $83.973(2)$ \\
\hline $\mathrm{O} 1^{\mathrm{ix}}-\mathrm{Na} 1-\mathrm{O} 1^{\mathrm{x}}$ & $136.88(3)$ & $\mathrm{Na} 1^{\mathrm{xiv}}-\mathrm{Na} 1-\mathrm{Na} 1^{\mathrm{xv}}$ & $123.539(1)$ \\
\hline $\mathrm{O} 1^{\mathrm{iv}}-\mathrm{Na} 1-\mathrm{Na} 1^{\text {xii }}$ & $40.383(14)$ & $\mathrm{Tc} 1-\mathrm{O} 1-\mathrm{Na} 1^{\mathrm{vii}}$ & $138.27(3)$ \\
\hline $\mathrm{O} 1^{\mathrm{v}}-\mathrm{Na} 1-\mathrm{Na} 1^{\mathrm{xii}}$ & $163.325(14)$ & $\mathrm{Tc} 1-\mathrm{O} 1-\mathrm{Na} 1^{\mathrm{xi}}$ & $118.74(3)$ \\
\hline 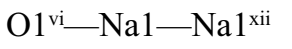 & $102.250(15)$ & $\mathrm{Na} 1^{\mathrm{vii}}-\mathrm{O} 1-\mathrm{Na} 1^{\mathrm{xi}}$ & $100.96(2)$ \\
\hline $\mathrm{O} 1^{\mathrm{vii}}-\mathrm{Na} 1-\mathrm{Na} 1^{\mathrm{xii}}$ & $65.896(14)$ & & \\
\hline
\end{tabular}

Symmetry codes: (i) $-y+5 / 4, x+1 / 4,-z+1 / 4$; (ii) $-x+1,-y+3 / 2, z$; (iii) $y-1 / 4,-x+5 / 4,-z+1 / 4$; (iv) $x-1, y-1 / 2,-z$; (v) $y-3 / 4,-x+5 / 4, z+1 / 4$; (vi) $-y+3 / 4$, $x-3 / 4, z+1 / 4$; (vii) $-x+1,-y+1,-z$; (viii) $-y+1 / 4, x-3 / 4,-z+1 / 4$; (ix) $-x+1,-y+1 / 2, z$; (x) $x-1, y, z$; (xi) $x+1, y, z$; (xii) $-x,-y,-z$; (xiii) $-x+1 / 2,-y+1 / 2$, $-z+1 / 2$; (xiv) $-x,-y+1,-z ;(\mathrm{xv})-x-1 / 2,-y+1 / 2,-z+1 / 2$. 